\title{
On Hydromagnetic Modified Thermohaline Convection - an Energy Relationship
}

\author{
H.i Mohan* \\ Dept. of Mathematics, International Centre for Distance Education and Open Learning (ICDEOL), Himachal Pradesh University, \\ Summer Hill Shimla-171005 (HP), India.
}

Received 17 June 2011; Revised 1 August 2011; Accepted 15 July 2012

\begin{abstract}
The problem of modified thermohaline magnetoconvection is considered in the present paper. An attempt is made to establish the relationship between various energies in Veronis' type configurations. The analysis made brings out that for Veronis type configuration the total kinetic energy associated with a disturbance exceeds the sum of its total magnetic and thermal energies in the parameter regime $\frac{Q \sigma_{1}}{\pi^{2}}+\frac{R_{S}^{\prime} \sigma}{\frac{27}{4} \tau^{2} \pi^{4}} \leq 1$. A similar energy relationship for Stern's type
\end{abstract} configuration is also established. Further, these results are valid for quite general nature of boundary conditions.

Keywords: DFIG, Wavelet, Time-Frequency Localization, Transients.

\section{Introduction}

Thermohaline convection or more generally double diffusive convection has matured into a subject possessing fundamental departure from its counterpart, namely single diffusive convection, and is of direct relevance in the fields of oceanography, astrophysics, liminology and chemical engineering etc. For a broad and a recent view of the subject one may be referred to Brandt and Fernando [1], Balmforth et al. [2], Malashetty et al. [3] and Huppert et al. [4]. Two fundamental configurations have been studied in the context of thermohaline instability problem, the first one by Stern [5] wherein the temperature gradient is stabilizing and the concentration gradient is destabilizing and the second one by Veronis [6] wherein the gradient is destabilizing and the concentration gradient is stabilizing. The main results derived by Stern and Veronis for their respective configurations are that both allow the occurrence of a stationary pattern of motions or oscillatory motions of growing amplitude provided the destabilizing concentration gradient or the temperature gradient is sufficiently large. However, stationary pattern of motion is the preferred mode of setting in of instability in case of Stern's configuration whereas oscillatory motions of growing amplitude are preferred in Veronis' configuration. More complicated double-diffusive phenomenon appears if the destabilizing thermal/concentration gradient is opposed by the effect of magnetic field or rotation.

Banerjee et al [7] presented a modified analysis of thermal and thermohaline instability of a liquid layer heated underside by emphasizing and utilizing the point that linear

\footnotetext{
* E-mail address: hm_math_hpu@rediffmail.com ISSN: 1791-237 7 @ 2012 Kavala Institute of Technology. All rights reserved.
}

theoretical explanation of the phenomenon of gravity dominated thermal instability in a liquid layer heated underside (Benard convection) should depend not only upon the Rayliegh number which is proportional to the uniform temperature difference maintained across the layer but also upon other parameter so that a provision could be made in the theory to recognize the fact that a relatively hotter layer with its heat diffusivity apparently increased/decreased as a consequence of an actual decreased/increased (depending on the fluid) in its specific heat at constant volume must exhibit Benard convection at a higher/lower Rayliegh number than a cooler layer under almost identical condition otherwise and further this qualitative effect is not quantitatively insignificant.

Chandrasekhar [8] in his investigation of magneto hydrodynamic simple Benard convection problem sought unsuccessfully the regime in terms of the parameters of the system alone, in which the total kinetic energy associated with a disturbance exceeds the total magnetic energy associated with it, since these considerations are of decisive significance in deciding the validity of the principle of exchange of stabilities. However, the solution for w ( = cons $\tan t(\sin \pi z))$ is not correct mathematically (and Chandrasekhar was aware of it). Banerjee et. al. until 1985 did not pursue their investigation in this direction and consequently did not see this connection. This gap in the literature on magnetoconvection has been completed by Banerjee et. al. [9] who presented a simple mathematical proof to establish that Chandrasekhar's conjecture is valid in the regime $Q \sigma_{1} \leq \pi^{2}$ and further this result is uniformly applicable for any combination of a dynamically free or rigid boundary when the region outside the liquid are perfectly conducting or insulating. Banerjee et al. [9] showed that in 
the parameter regime $\frac{Q \sigma_{1}}{\pi^{2}} \leq 1$ the total kinetic energy associated with a disturbance is greater than the total magnetic energy associated with it.

Banerjee et al. [10] further extended these energy considerations to a more general problem, namely, magnetohydrodynamic thermohaline convection problem, of Stern's type and established that in the parameter regime $\frac{Q \sigma_{1}}{\pi^{2}}+\frac{|R| \sigma}{\pi^{4}} \leq 1$, the total kinetic energy associated with a disturbance exceeds the sum of its total magnetic and thermal energies. A similar characterization theorem in magnetothermohaline convection of the Veronis' type was also established by Banerjee et. al in the subsequent year. Mohan et al. [11] derived a characterization theorem in hydromagnetic double diffusive convection and established that the total kinetic energy associated with a disturbance is greater than the sum of its total magnetic and concentration energies in the parameter regime $\frac{Q \sigma_{1}}{\pi^{2}}+\frac{R_{S} \sigma}{4 \tau^{2} \pi^{4}} \leq 1$. Recently, Mohan [12] extended these results to the problems of double-diffusive convection coupled with crossdiffusions.

The present analysis extends these energies considerations to the hydromagnetic modified thermohaline convection problem of Veronis'and Stern's type configurations. The nature of system of equations for the present problem is clearly qualitatively different from those of thermohaline convection problem and the results are obviously not derivable by the method adopted by Banerjee et.al and Mohan et.al.in this direction on account of nontrivial coupling between $\theta, \phi$ and $w$ in the equation of heat conduction. However, a close and critical look at derivation of this equation makes one feel that this difficulty can be taken care of by an appropriate transformation. The aim of the present paper is to construct such a transformation which overcomes the above difficulty and enables us to derive the desired energy relationship in the present modified set up.

\section{Mathematical Formulation and Analysis}

Following Banerjee et al. [7], the relevant governing equations and boundary conditions of the modified thermohaline instability in their non-dimensional form are given by:

$$
\begin{aligned}
& \left(D^{2}-a^{2}\right)\left(D^{2}-a^{2}-\frac{p}{\sigma}\right) w= \\
& R_{T} a^{2} \theta-R_{S} a^{2} \phi-Q D\left(D^{2}-a^{2}\right) h_{z} \\
& \left(D^{2}-a^{2}-p\left\langle 1-T_{0} \alpha_{2}\right\rangle\right) \theta-T_{0} \hat{\alpha}_{2} R_{3} p \phi= \\
& -\left(1-T_{0} \alpha_{2}\right) w-T_{0} \hat{\alpha}_{2} R_{3} w \\
& \left(D^{2}-a^{2}-\frac{p}{\tau}\right) \phi=-\frac{w}{\tau}
\end{aligned}
$$

and

$$
\left(D^{2}-a^{2}-\frac{p \sigma_{1}}{\sigma}\right) h_{z}=-D w
$$

sith $w=0=\theta=\phi$ on both the boundaries, $D^{2} w=0$ on a tangent stress-free boundary everywhere, $D w=0$ on a rigid boundary, $h_{z}=0$ on both the boundaries if the regions outside the fluid are perfectly conducting,

$$
\left.\begin{array}{l}
D h_{z}=-a h_{z} \text { at } z=1 \\
D h_{z}=a h_{z} \text { at } z=0
\end{array}\right\} \text { if the regions outside the fluid are }
$$$$
\text { insulating }
$$

The meanings of symbols from physical point of view are as follows: $\mathrm{z}$ is the vertical coordinate, $\mathrm{d} / \mathrm{dz}$ is differentiation along the vertical direction, $\mathrm{a}^{2}$ is square of horizontal wave number, $\sigma=\frac{v}{\kappa}$ is the thermal Prandtl number, $\sigma_{1}=\frac{v}{\eta}$ is the magnetic Prandtl number, $\tau=\frac{\eta_{1}}{\kappa}$ is the Lewis number, $R_{T}=\frac{g \alpha \beta_{1} d^{4}}{\kappa v}$ is the thermal Rayleigh number, $R_{S}=\frac{g \alpha \beta_{2} d^{4}}{\kappa v}$ is the concentration Rayleigh number, $Q=\frac{\mu^{2} H^{2} \sigma d^{2}}{\rho v}$ is the Chandrasekhar number, w is the vertical velocity, $\theta$ is the temperature, $\phi$ is the concentration, $\mathrm{p}$ is the complex growth rate, $\alpha_{2}$ is the coefficient of specific heat due to variation in temperature and $\hat{\alpha}_{2}$ is analogous coefficient due to variation in concentration, $h_{z}$ is the vertical magnetic field.

In (1)-(5), $\mathrm{z}$ is real independent variable such that $0 \leq \mathrm{z} \leq 1, D=\frac{d}{d z}$ is differentiation w.r.t $\mathrm{z}, \mathrm{a}^{2}$ is a constant, $\sigma>0$ is a constant, $\sigma_{1}>0$ is a constant, $\tau>0$ is a constant, $R_{T}$ and $\mathrm{R}_{\mathrm{S}}$ are positive constants for the Veronis' configuration and negative constants for Stern's configuration, $R_{3}=\frac{\beta^{\prime}}{\beta}$ is the ratio of concentration gradient to thermal gradient, $\mathrm{p}=\mathrm{p}_{\mathrm{r}}+\mathrm{i} \mathrm{p}_{\mathrm{i}}$ is complex constant in general such that $p_{r}$ and $p_{i}$ are real constants and as a consequence the dependent variables $\mathrm{w}(\mathrm{z})=\mathrm{w}_{\mathrm{r}}(\mathrm{z})+\mathrm{iw}_{\mathrm{i}}(\mathrm{z})$, $\theta(\mathrm{z})=\theta_{r}(\mathrm{z})+i \theta_{i}(\mathrm{z})$ and $\phi(\mathrm{z})=\phi_{r}(\mathrm{z})+i \phi_{i}(\mathrm{z})$ are complex valued functions(and their real and imaginary parts are real valued).

We now prove the following theorems:

Theorem 1: If ( $\mathrm{p}, \mathrm{w}, \theta, \phi, \mathrm{h}_{\mathrm{z}}$ ), $\mathrm{p}=\mathrm{p}_{\mathrm{r}}+\mathrm{ip}_{\mathrm{i}}, \mathrm{p}_{\mathrm{r}} \geq 0$ is a solution of (1) - (4) together with boundary conditions (5) with $R_{T}>0 R_{S}>0$ and $\frac{Q \sigma_{1}}{\pi^{2}}+\frac{R_{S}^{\prime} \sigma}{\frac{27}{4} \tau^{2} \pi^{4}} \leq 1$, then

$\int_{0}^{1}\left(|D w|^{2}+a^{2}|w|^{2}\right) d z>Q \sigma_{1} \int_{0}^{1}\left(\left|D h_{z}\right|^{2}+a^{2}\left|h_{z}\right|^{2}\right) d z+R_{S}^{\prime} a^{2} \sigma \int_{0}^{1}|\phi|^{2} d z$

Proof: Equation (2) upon utilizing (3) can be written as

$$
\begin{aligned}
& \left(D^{2}-a^{2}-p\left\langle 1-T_{0} \alpha_{2}\right\rangle\right) \theta-T_{0} \hat{\alpha}_{2} R_{3} p \tau\left(D^{2}-a^{2}\right) \phi= \\
& -\left(1-T_{0} \alpha_{2}\right) w
\end{aligned}
$$


Using the transformations

$\mathscr{W}=w$

$\theta=\frac{\left\langle\tau\left(1-T_{0} \alpha_{2}\right)-1\right\rangle}{T_{0} \hat{\alpha}_{2} R_{3} \tau} \theta+\phi$

$\phi$

$h_{z}=h_{z}$

Equations (1), (3), (4) and (6) and the associated boundary conditions (5) assume the following forms:

$$
\begin{aligned}
& \left(D^{2}-a^{2}\right)\left(D^{2}-a^{2}-\frac{p}{\sigma}\right) w= \\
& R_{T}^{\prime} a^{2} \theta-R_{S}^{\prime} a^{2} \phi-Q D\left(D^{2}-a^{2}\right) h_{z} \\
& \left\{D^{2}-a^{2}-p\left(1-T_{0} \alpha_{2}\right)\right\} \theta=-B w \\
& \left(D^{2}-a^{2}-\frac{p}{\tau}\right) \phi=-\frac{w}{\tau} \\
& \left(D^{2}-a^{2}-\frac{p \sigma_{1}}{\sigma}\right) h_{z}=-D w
\end{aligned}
$$

with $w=0=\theta=\phi$ on both the boundaries, $D^{2} w=0$ on a tangent stress-free boundary everywhere, $D w=0$ on a rigid boundary, $h_{z}=0$ on both the boundaries if the regions outside the fluid are perfectly conducting,

$\left.\begin{array}{l}D h_{z}=-a h_{z} \text { at } z=1 \\ D h_{z}=a h_{z} \text { at } z=0\end{array}\right\}$ if the regions outside the fluid are insulating,

where $R_{T}^{\prime}=\frac{R_{T} T_{0} \hat{\alpha}_{2} R_{3} \tau}{\left\langle\tau\left(1-T_{0} \alpha_{2}\right)-1\right\rangle}, R_{S}^{\prime}=R_{S}+\frac{R_{T} T_{0} \hat{\alpha}_{2} R_{3} \tau}{\left\langle\tau\left(1-T_{0} \alpha_{2}\right)-1\right\rangle}$,

$\mathrm{B}=\left(1-T_{0} \alpha_{2}\right)\left\{1+\frac{\left(\tau\left\langle 1-T_{0} \alpha_{2}\right\rangle-1\right)}{T_{0} \hat{\alpha}_{2} R_{3} \tau}\right\}>0$ and the symbol $\sim$ has been omitted for convenience.

Multiplying (11) by $h_{z}^{*}$ (the complex conjugate of $h_{z}$ ), integrating the resulting equation over the range of $z$ by parts a suitable number of times, and making use of the boundary conditions (12) we get

$a M+\int_{0}^{1}\left(\left|D h_{z}\right|^{2}+a^{2}\left|h_{z}\right|^{2}\right) d z+\frac{p \sigma_{1}}{\sigma} \int_{0}^{1}\left|h_{z}\right|^{2} d z=-\int_{0}^{1} w D h_{z}^{*}$

where $M=\left\{\left(\left|h_{z}\right|^{2}\right)_{0}+\left(\left|h_{z}\right|^{2}\right)_{1}\right\} \geq 0$.

Equating the real part of (13), we get

$$
a M+\int_{0}^{1}\left(\left|D h_{z}\right|^{2}+a^{2}\left|h_{z}\right|^{2}\right) d z+\frac{p_{r} \sigma_{1}}{\sigma} \int_{0}^{1}\left|h_{z}\right|^{2} d z
$$

$$
\begin{aligned}
& =\operatorname{Re} a \text { l part of }\left(-\int_{0}^{1} w D h_{z}^{*} d z\right) \\
& \leq\left|\int_{0}^{1} w D h_{z}^{*} d z\right| \\
& \leq \int_{0}^{1}|w|\left|D h_{z}\right| d z \\
& \leq\left\{\int_{0}^{1}|w|^{2} d z\right\}^{1 / 2}\left\{\int_{0}^{1}\left|D h_{z}\right|^{2} d z\right\}^{1 / 2} \\
& \text { (using Schwartz inequality) }
\end{aligned}
$$

Since $\mathrm{p}_{\mathrm{r}} \geq 0$, therefore from (14), we get

$$
\int_{0}^{1}\left|D h_{z}\right|^{2} d z<\left\{\int_{0}^{1}|w|^{2} d z\right\}^{1 / 2}\left\{\int_{0}^{1}\left|D h_{z}\right|^{2} d z\right\}^{1 / 2}
$$

or

$$
\int_{0}^{1}\left|D h_{z}\right|^{2} d z<\int_{0}^{1}|w|^{2} d z
$$

Using (15), it follows from (14) that

$$
\int_{0}^{1}\left(\left|D h_{z}\right|^{2}+a^{2}\left|h_{z}\right|^{2}\right) d z<\int_{0}^{1}|w|^{2} d z
$$

Since $\mathrm{w}(0)=0=\mathrm{w}$ (1), therefore using Rayleigh-Ritz inequality [13], we get

$$
\int_{0}^{1}|w|^{2} d z<\frac{1}{\pi^{2}} \int_{0}^{1}|D w|^{2} d z
$$

It follows from (16) and (17) that

$$
\int_{0}^{1}\left(\left|D h_{z}\right|^{2}+a^{2}\left|h_{z}\right|^{2}\right) d z<\frac{1}{\pi^{2}} \int_{0}^{1}|D w|^{2} d z<\frac{1}{\pi^{2}} \int_{0}^{1}\left(|D w|^{2}+a^{2}|w|^{2}\right) d z
$$

$$
\begin{aligned}
& \text { or } \\
& \begin{aligned}
Q \sigma_{1} \int_{0}^{1}\left(\left|D h_{z}\right|^{2}+a^{2}\left|h_{z}\right|^{2}\right) d z+R_{s}^{\prime} \sigma a^{2} \int_{0}^{1}|\phi|^{2} d z \\
<\frac{Q \sigma_{1}}{\pi^{2}} \int_{0}^{1}\left(|D w|^{2}+a^{2}|w|^{2}\right) d z+R_{s}^{\prime} \sigma a^{2} \int_{0}^{1}|\phi|^{2} d z(18)
\end{aligned}
\end{aligned}
$$

Multiplying (10) by the complex conjugate of (10) and integrating by parts over the vertical range of $\mathrm{z}$ for an appropriate number of times and making use of the boundary conditions (12) we get

$$
\begin{aligned}
& \int_{0}^{1}\left(\left|D^{2} \phi\right|^{2}+2 a^{2}|D \phi|^{2}+a^{4}|\phi|^{2}\right) d z+2 p_{r} \int_{0}^{1}\left(|D \phi|^{2}+a^{2}|\phi|^{2}\right) d z \\
& +\frac{|p|^{2}}{\tau^{2}} \int_{0}^{1}|\phi|^{2} d z=\frac{1}{\tau^{2}} \int_{0}^{1}|w|^{2} d z
\end{aligned}
$$

Since, $p_{r} \geq 0$, therefore, from (19), we get 


$$
\int_{0}^{1}\left(\left|D^{2} \phi\right|^{2}+2 a^{2}|D \phi|^{2}+a^{4}|\phi|^{2}\right) d z<\frac{1}{\tau^{2}} \int_{0}^{1}|w|^{2} d z
$$

Since $\phi(0)=0=\phi(1)$, therefore using Rayleigh-Ritz inequality [13], we get

$$
\pi^{2} \int_{0}^{1}|\phi|^{2} d z<\int_{0}^{1}|D \phi|^{2} d z
$$

and also

$$
\pi^{4} \int_{0}^{1}|\phi|^{2} d z \leq \int_{0}^{1}\left|D^{2} \phi\right|^{2} d z \text { (using Schwartz inequality) }
$$

It follows from (20) and (21) that

$$
\left(\pi^{2}+a^{2}\right)^{2} \int_{0}^{1}|\phi|^{2} d z<\frac{1}{\tau^{2}} \int_{0}^{1}|w|^{2} d z
$$

or

$$
\begin{aligned}
& \frac{\left(\pi^{2}+a^{2}\right)^{2}}{a^{2}} \int_{0}^{1}|\phi|^{2} d z<\frac{1}{a^{2} \tau^{2}} \int_{0}^{1}|w|^{2} d z< \\
& \frac{1}{a^{2} \tau^{2}\left(\pi^{2}+a^{2}\right)} \int_{0}^{1}\left(|D w|^{2}+a^{2}|w|^{2}\right) d z
\end{aligned}
$$$$
\text { or } a^{2} \int_{0}^{1}|\phi|^{2} d z<\frac{1}{\frac{27}{4} \pi^{4} \tau^{2}} \int_{0}^{1}\left(|D w|^{2}+a^{2}|w|^{2}\right) d z,
$$

since the minimum value of $\frac{\left(\pi^{2}+a^{2}\right)^{3}}{a^{2}}$ for $\mathrm{a}^{2}>0$ is $\frac{27 \pi^{4}}{4}$

or

$$
R_{s}^{\prime} a^{2} \sigma \int_{0}^{1}|\phi|^{2} d z<\frac{R_{s}^{\prime} \sigma}{\frac{27}{4} \pi^{4} \tau^{2}} \int_{0}^{1}\left(|D w|^{2}+a^{2}|w|^{2}\right) d z
$$

Now from (18) and (22), we get

$$
\begin{aligned}
& Q \sigma_{1} \int_{0}^{1}\left(\left|D h_{z}\right|^{2}+a^{2}\left|h_{z}\right|^{2}\right) d z+R_{s}^{\prime} a^{2} \sigma \int_{0}^{1}|\phi|^{2} d z \\
& <\left(\frac{Q \sigma_{1}}{\pi^{2}}+\frac{R_{s}^{\prime} \sigma}{\frac{27}{4} \tau^{2} \pi^{4}}\right)_{0}^{1}\left(|D w|^{2}+a^{2}|w|^{2}\right) d z
\end{aligned}
$$

Therefore, if $\frac{Q \sigma_{1}}{\pi^{2}}+\frac{R_{s}^{\prime} \sigma}{\frac{27}{4} \tau^{2} \pi^{4}} \leq 1$, then from (23), we get

$$
\begin{aligned}
& \int_{0}^{1}\left(|D w|^{2}+a^{2}|w|^{2}\right) d z>Q \sigma_{1} \int_{0}^{1}\left(\left|D h_{z}\right|^{2}+a^{2}\left|h_{z}\right|^{2}\right) d z \\
& +R_{s}^{\prime} a^{2} \sigma \int_{0}^{1}|\phi|^{2} d z
\end{aligned}
$$

and this completes the proof of the theorem.

We note that the left hand side of (24) represents the total kinetic energy associated with a disturbance while the right hand side represents the sum of its total magnetic and concentration energies, and Theorem 1 may be stated in the following equivalent form:

At the neutral or unstable state in the hydromagnetic modified thermohaline convection problem of the Veronis' type configuration, the total kinetic energy associated with a disturbance is greater than the sum of its total magnetic and concentration energies in the parameter regime $\frac{Q \sigma_{1}}{\pi^{2}}+\frac{R_{s}^{\prime} \sigma}{\frac{27}{4} \tau^{2} \pi^{4}} \leq 1$ and this result is uniformly valid for any combination of dynamically free or rigid boundaries that are either perfectly conducting or insulating.

Theorem 2: If ( $\left.\mathrm{p}, \mathrm{w}, \theta, \phi, h_{z}\right), \mathrm{p}=\mathrm{p}_{\mathrm{r}}+\mathrm{i} \mathrm{p}_{\mathrm{i}}, \mathrm{p}_{\mathrm{r}} \geq 0$ is a solution of $(8)-(11)$ together with boundary conditions (12) with $R_{T}<0, \mathrm{R}_{\mathrm{S}}<0$, and $\frac{Q \sigma_{1}}{\pi^{2}}+\frac{\left|R_{T}^{\prime}\right| \sigma B^{2}}{\frac{27}{4} \pi^{4}} \leq 1$ then

$$
\begin{aligned}
& \int_{0}^{1}\left(|D w|^{2}+a^{2}|w|^{2}\right) d z>Q \sigma_{1} \int_{0}^{1}\left(\left|D h_{z}\right|^{2}+a^{2}\left|h_{z}\right|^{2}\right) d z \\
& +\left|R_{T}{ }^{\prime}\right| a^{2} \sigma \int_{0}^{1}|\theta|^{2} d z
\end{aligned}
$$

Proof: Putting $R_{T}^{\prime}=-\left|R_{T}^{\prime}\right|$ and $R_{S}^{\prime}=-\left|R_{S}^{\prime}\right|$, (8) in the present case assume the following form:

$$
\begin{aligned}
& \left(D^{2}-a^{2}\right)\left(D^{2}-a^{2}-\frac{p}{\sigma}\right) w= \\
& -\left|R_{T}{ }^{\prime}\right| a^{2} \theta+\left|R_{S}{ }^{\prime}\right| a^{2} \phi-Q D\left(D^{2}-a^{2}\right) h_{z}
\end{aligned}
$$

Proceeding exactly as in Theorem 1, (18) in the present case can be written as

$$
\begin{aligned}
& Q \sigma_{1} \int_{0}^{1}\left(\left|D h_{z}\right|^{2}+a^{2}\left|h_{z}\right|^{2}\right) d z+\left|R_{T}^{\prime}\right| \sigma a^{2} \int_{0}^{1}|\theta|^{2} d z \\
& <\frac{Q \sigma_{1}}{\pi^{2}} \int_{0}^{1}\left(|D w|^{2}+a^{2}|w|^{2}\right) d z+\left|R_{T}^{\prime}\right| \sigma a^{2} \int_{0}^{1}|\phi|^{2} d z
\end{aligned}
$$

Multiplying (9) by the complex conjugate of (9) and integrating by parts over the vertical range of $z$ for an appropriate number of times and making use of the boundary conditions (12) we get 


$$
\begin{aligned}
& \int_{0}^{1}\left(\left|D^{2} \theta\right|^{2}+2 a^{2}|D \theta|^{2}+a^{4}|\theta|^{2}\right) d z \\
& +2 p_{r}\left(1-T_{0} \alpha_{2}\right) \int_{0}^{1}\left(|D \theta|^{2}+a^{2}|\theta|^{2}\right) d z \\
& +|p|^{2}\left(1-T_{0} \alpha_{2}\right)^{2} \int_{0}^{1}|\theta|^{2} d z=B^{2} \int_{0}^{1}|w|^{2} d z
\end{aligned}
$$

Since, $\mathrm{p}_{\mathrm{r}} \geq 0$, therefore from (28), we get

$$
\int_{0}^{1}\left(\left|D^{2} \theta\right|^{2}+2 a^{2}|D \theta|^{2}+a^{4}|\theta|^{2}\right) d z<B^{2} \int_{0}^{1}|w|^{2} d z
$$

Since $\theta(0)=0=\theta(1)$, therefore using Rayleigh-Ritz inequality [9], we get

$$
\pi^{2} \int_{0}^{1}|\theta|^{2} d z<\int_{0}^{1}|D \theta|^{2} d z
$$

and also

$$
\pi^{4} \int_{0}^{1}|\theta|^{2} d z \leq \int_{0}^{1}\left|D^{2} \theta\right|^{2} d z \text { (using Schwartz inequality) }
$$

It follows from (29) and (30) that

$$
\left(\pi^{2}+a^{2}\right)^{2} \int_{0}^{1}|\theta|^{2} d z<B^{2} \int_{0}^{1}|w|^{2} d z
$$

or

$$
\begin{aligned}
& \frac{\left(\pi^{2}+a^{2}\right)^{2}}{a^{2}} \int_{0}^{1}|\theta|^{2} d z<\frac{B^{2}}{a^{2}} \int_{0}^{1}|w|^{2} d z< \\
& \frac{B^{2}}{a^{2}\left(\pi^{2}+a^{2}\right)} \int_{0}^{1}\left(|D w|^{2}+a^{2}|w|^{2}\right) d z
\end{aligned}
$$

or

$$
a^{2} \int_{0}^{1}|\theta|^{2} d z<\frac{B^{2}}{\frac{27}{4} \pi^{4}} \int_{0}^{1}\left(|D w|^{2}+a^{2}|w|^{2}\right) d z
$$

since the minimum value of $\frac{\left(\pi^{2}+a^{2}\right)^{3}}{a^{2}}$ for $\mathrm{a}^{2}>0$ is $\frac{27 \pi^{4}}{4}$. or

$$
\left|R_{T}^{\prime}\right| a^{2} \sigma \int_{0}^{1}|\theta|^{2} d z<\frac{\left|R_{T}^{\prime}\right| B^{2} \sigma}{\frac{27}{4} \pi^{4}} \int_{0}^{1}\left(|D w|^{2}+a^{2}|w|^{2}\right) d z
$$

Now from (27) and (31), we get

$$
\begin{aligned}
& Q \sigma_{1} \int_{0}^{1}\left(\left|D h_{z}\right|^{2}+a^{2}\left|h_{z}\right|^{2}\right) d z+\left|R_{T}^{\prime}\right| a^{2} \sigma \int_{0}^{1}|\theta|^{2} d z \\
& <\left(\frac{Q \sigma_{1}}{\pi^{2}}+\frac{\left|R_{T}^{\prime}\right| B^{2} \sigma}{\frac{27^{2}}{4} \pi^{4}} \int_{0}^{1}\left(|D w|^{2}+a^{2}|w|^{2}\right) d z\right.
\end{aligned}
$$

Therefore, if $\frac{Q \sigma_{1}}{\pi^{2}}+\frac{\left|R_{T}^{\prime}\right| B^{2} \sigma}{\frac{27}{4} \pi^{4}} \leq 1$, then from (32), we get

$$
\begin{aligned}
& \int_{0}^{1}\left(|D w|^{2}+a^{2}|w|^{2}\right) d z>Q \sigma_{1} \int_{0}^{1}\left(\left|D h_{z}\right|^{2}+a^{2}\left|h_{z}\right|^{2}\right) d z \\
& +\left|R_{T}^{\prime}\right| a^{2} \sigma \int_{0}^{1}|\theta|^{2} d z
\end{aligned}
$$

and this completes the proof of the theorem.

We note that the left hand side of (33) represents the total kinetic energy associated with a disturbance while the right hand side represents the sum of its total magnetic and thermal energies and Theorem 2 may be stated in the following equivalent form:

At the neutral or unstable state in the hydromagnetic modified thermohaline convection problem of the Stern's type configuration, the total kinetic energy associated with a disturbance is greater than the sum of its total magnetic and thermal energies in the parameter regime $\frac{Q \sigma_{1}}{\pi^{2}}+\frac{\left|R_{T}^{\prime}\right| B^{2} \sigma}{\frac{27}{4} \pi^{4}} \leq 1$ and this result is uniformly valid for any combination of dynamically free or rigid boundaries that are either perfectly conducting or insulating.

\section{Conclusions}

In the present paper, the hydromagnetic modified thermohaline convection problem of Veronis' and Stern's type configuration is considered. The analysis made brings out the following main conclusions:

i. At the neutral or unstable state in the magnetohydrodynamic thermohaline convection problem of the Veronis' type configuration, the total kinetic energy associated with a disturbance is greater than the sum of its total magnetic and concentration energies in the parameter regime $\frac{Q \sigma_{1}}{\pi^{2}}+\frac{R_{S}^{\prime} \sigma}{\frac{27}{4} \tau^{2} \pi^{4}} \leq 1$ and this result is uniformly valid for any combination of dynamically free or rigid boundaries that are either perfectly conducting or insulating.

ii. At the neutral or unstable state in the hydromagnetic modified thermohaline convection problem of the Stern's type configuration, the total kinetic energy associated with a disturbance is greater than sum of its total magnetic and thermal energies in the parameter regime $\frac{Q \sigma_{1}}{\pi^{2}}+\frac{\left|R_{T}^{\prime}\right| \sigma}{\frac{27}{4} \pi^{4}} \leq 1$ and this result is uniformly valid for any combination of dynamically free or rigid boundaries that are either perfectly conducting or insulating. 


\section{References}

1. A. Brandt and H.J.S. Fernando, American Geophysical Union (1996).

2. N. J. Balmforth, S. A. Ghadge, A. Kettapum and S.D. Mandre, J. Fluid Mech. 569, 29 (2006).

3. M. S. Malashetty and B. S. Biradar, Phys. Fluids 23, doi: 10. 1063/1.3601482, 2011.

4. E.H. Huppert and J. S. Turner, J. Fluid Mech. 106 (2006) 299.

5. M .E. Stern, Tellus, 12 (1960) 172.

6. G. Veronis, J.Mars. Res., 1 (1965) 23.

7. M.B. Banerjee, J.R. Gupta, R.G. Shandil, K.C. Sharma and D.C. Katoch, .Math.Phy.Sci. 17 (1983) 603.
8. S.Chandrasekhar, Philos. Mag. 43, 501 (1952).

9. M.B. Banerjee and S.P Katyal, J. Math. Anal. Appl. 129383 (1988).

10. M.B. Banerjee, J.R. Gupta and S.P. Katyal, Indian J. Pure Appl.Math. 18(9) (1987) 865.

11. H. Mohan, P. Kumar and P. Devi, Ganita. 57(2) (2006) 149.

12. H. Mohan, Studia Geo. Tech. et Mech. XXXII, 23 (2010).

13. M.H Schultz, Spline Analysis, Prentice-Hall, Englewood Cliffs, N.J. (1973). 